\title{
Philosophical Foundations of Effective Field Theories
}

\author{
Sébastien Rivat* and Alexei Grinbaum ${ }^{\dagger}$
}

July 26, 2019

\begin{abstract}
This survey covers some of the main philosophical debates raised by the framework of effective field theories during the last decades. It is centered on three issues: whether effective field theories underpin a specific realist picture of the world, whether they support an antireductionist picture of physics, and whether they provide reasons to give up the ultimate aspiration of formulating a final and complete physical theory. Reviewing the past and current literature, we argue that effective field theories do not give convincing reasons to adopt a particular stance towards these speculative issues. They hold good prospects for asking ontologically perspicuous and sensible questions about currently accessible domains. With respect to more fundamental questions, however, the only certainty is provisional and instrumental: effective theories are currently indispensable for conducting fruitful scientific research.
\end{abstract}

\section{What is an effective field theory?}

The term effective has a variety of meanings in exact sciences. A computational method is effective if it requires only a reasonable amount of time and resources. An effective prediction is one that has high success rate and decisive value for evaluating some hypothesis. We review various philosophical issues related to one particular meaning of the term, namely a set of characteristics which distinguish a specific type of theories, called Effective Field Theories (EFTs), from other traditional products of scientific theorizing, such as phenomenological models or putatively fundamental theories [27]. Although this meaning of the term effective might appear restricted, the framework of EFTs is actually quite extensive. It is closely associated with a variety of instruments and results, including the modern Wilsonian Renormalization Group (RG) theory and lattice methods in Quantum Field

*Department of Philosophy, Columbia University, New York, USA

${ }^{\dagger}$ CEA-Saclay, IRFU/Larsim, 91191 Gif-sur-Yvette Cedex, France 
Theory (QFT). This framework is also successfully implemented in various disciplines ranging from nuclear physics to inflationary cosmology and applied to different types of classical and quantum theories as well as point-particle and low-energy string theories. This large success suggests that EFTs might significantly impact current and future philosophical discussions about physics. Before engaging with the debates that have animated the literature in the last decades, we first set the stage with a few conceptual and methodological remarks.

While EFTs became increasingly widespread in the last forty years, they bear some conceptual affinity with earlier research programs [43, 44]. In the 1950s, the physics community was not sure whether the methods of QFT based on the use of gauge symmetry would lead one to a correct understanding of the strong interaction. Similar doubts had been expressed and successfully lifted a decade earlier with regard to quantum electrodynamics. However, the strong interaction did not seem to yield to the same methods and give way to a similar solution. In 1954, at a conference attended by Oppenheimer, Gell-Mann, Fermi, Wick, and Dyson, the physicist Goldberger challenged the applicability of QFT to nuclear interactions. Surprisingly, nobody objected to the contrary [34]. The reason had to do with the irreducible divergencies that arise in the application of perturbative methods to theories with strong couplings. Around this period, physicists began to look for a non-perturbative framework to treat the strong interaction and they needed a new methodology. One of the best known answers was to be found in the $S$-matrix approach, which had already been impulsed in the 1940s through the work of Heisenberg. The $S$-matrix only aimed at asking questions about physical observables: it avoided the need to have knowledge about the fundamental structure of interactions between elementary particles [26].

In the case of EFTs, the unknown element can be new physics beyond the Standard Model or the exact structure of interactions between quarks and gluons at low energies. In analogy with the $S$-matrix approach, EFTs aim at asking questions about the physics relevant within a specific domain, without the need to worry about the physical content of the fundamental theory. Just as the $S$-matrix enables an exclusive focus on observable quantities by disregarding the quantum field, EFTs free the theorist from the need to worry about the particular details of high-energy physics. To this end, the EFT methodology prescribes that the Lagrangians include all the possible terms compatible with a given set of symmetry constraints. If the high-energy theory is unknown, the hope is that all high-energy effects on low-energy physics can be accounted for by such terms. If, on the contrary, the high-energy theory is known, then high-energy physics typically generates the most general low-energy Lagrangian compatible with the symmetries of the theory.

Consider first an abstract example of EFT. The theory is typically defined by a Lagrangian valid up to some scale $\Lambda$, which divides into a finite sum of local operators of dimension four or less and an infinite sum of higher dimensional local operators with dimensionless coupling 
parameters $\lambda_{n}$ :

$$
\mathcal{L}=\mathcal{L}^{(d \leq 4)}+\sum_{n=1}^{\infty} \frac{\lambda_{n}}{\Lambda^{n}} \mathcal{O}_{n} .
$$

In general, the value of the parameters of $\mathcal{L}$ is either fixed experimentally (at least for a finite number of them) or determined from the underlying high-energy theory through RG calculations, and the form of the operators $\mathcal{O}_{n}$ is fixed by the symmetries of the theory. The parameters of $\mathcal{L}^{(d \leq 4)}$ and the higher order terms encode information about the unknown physics at scales higher than $\Lambda$, which means that this Lagrangian can be used to study low-energy effects of unknown high-energy physics without having to specify what this physics actually is.

As a concrete realistic example, consider the electroweak EFT without the Higgs field, which reproduces the Standard Model for the light degrees of freedom (light quarks, leptons and electroweak gauge bosons) at sufficiently small energies compared to the Higgs mass [35]. The lowest-order effective Lagrangian $\mathcal{L}_{\mathrm{EW}}^{(d<4)}$ includes the standard kinetic terms for the electroweak gauge bosons, the leptons, the quarks, and the Goldstone bosons. At the fourth order, the most general effective Lagrangian with only gauge bosons and Goldstone bosons,

$$
\mathcal{L}_{\mathrm{EW}}^{(4)}=\sum_{i=0}^{14} a_{i} \mathcal{O}_{i},
$$

contains fifteen operators. Again, this complexity stems from the requirement that we use the most general form of the Lagrangian compatible with symmetry principles.

Gell-Mann once formulated a rule called "the totalitarian principle", which asserts that everything that is not forbidden is compulsory [20]. The EFT Lagrangians (1) and (2) perfectly illustrate this rule. If one wishes to offer the most complete description of some low-energy phenomena by using only a few symmetry principles, then the most coherent, economical, and procedurally concise prescription would be to include all possible terms consistent with such principles. The resulting Lagrangian has a complex structure but the inclusion of multiple interaction terms is precisely what makes EFTs so attractive (to turn Gell-Mann's political metaphor upside down, it is democratic inclusivity that leads to felicitous complexity). Through this procedure, the theorist is able to parametrize any high-energy effect in accordance with its relevance at low energies and systematically organize potential information about unknown physics. The structure of the EFT Lagrangian also enables the theorist to compute correction terms by increasing order in the inverse power of the scale $\Lambda$, thereby extracting highly precise predictions.

Taking the complexity of a theory to be one of its chief virtues is a far cry from traditional remarks made both by philosophers and physicists. Quine named several criteria of theory selection without realizing that they may come in conflict with one another: "Simplicity, 
economy and naturalness contribute to the molding of scientific theories generally" [37]. Dirac also praised simplicity, although he ranked it after mathematical beauty:

The research worker, in his efforts to express the laws of Nature in mathematical form, should strive mainly for mathematical beauty. He should still take simplicity into consideration in a subordinate way to beauty. ... It often happens that the requirements of simplicity and beauty are the same, but when they clash the latter must take precedence. [14]

Perhaps most clearly of all, Wigner came to raise some doubts about the relevance of the criterion of simplicity in science. While physical theories are collections of laws of nature, mathematical concepts that express them are of our choosing. We "manipulate [them for] making striking, brilliant arguments" but do not select them for their simplicity [46]. Yet, Wigner was still far from praising the lack of simplicity as a correlate of the efficiency and predictive power of a theory.

To continue with our example, the structure of the EFT Lagrangian (2) is still carefully tailored despite its complexity. $C P$ and $S U(2)_{L} \times$ $U(1)_{Y}$ invariance impose strict symmetry constraints on the set of possible operators. Also, three of the fifteen operators can be eliminated by using the equations of motion under the assumption that the fermions are (approximately) massless. With the remaining terms, one finds various effects such as the usual electroweak oblique corrections, corrections to rare $B$ and $K$ decays, or the $C P$-violating parameter. And just like the $S$-matrix, the electroweak EFT allows one to ignore the dynamics of fields relevant at high energies, including the Higgs field, and therefore to offer a simplified description of the low-energy electroweak sector of the Standard Model.

This last point calls for a more general methodological remark. The electroweak EFT is actually developed by following a 'top-down' strategy: the high-energy physical theory is known but unsuitable to study the low-energy regime. This may occur for a variety of reasons: the computation is too complex, the perturbative method is inapplicable, or the high-energy theory does not provide enough physical details in the low-energy regime. Top-down EFTs were employed as early as the 1930s, e.g., in the Euler-Heisenberg calculation of photonphoton scattering at small energies within the framework of Dirac's QFT $[15,16,28]$. A more recent example is chiral perturbation theory, which gives a low-energy approximation of quantum chromodynamics in the light quark sector. Yet another example is the use of EFTs in condensed matter physics: even when the underlying theory is known, often the only tractable way to compute low-energy observables is to build an effective model as if the underlying theory were unknown.

Current high-energy physics also uses an alternative 'bottom-up' strategy: the underlying high-energy theory is unknown and the lowenergy EFT is constructed by formulating the most general Lagrangian compatible with a given set of degrees of freedom and symmetries. Theoretic constructs that employ fundamental principles tasked with limiting the possibilities in a description of unknown facts have been 
called "blackbox models" [26]. Although blackbox models appear in many fields of physics, from Einstein's "principle theories" to deviceindependent methods in quantum information [25], in the case of EFTs they take on a universal and fundamental character: it is common among physicists to think of all current physical theories, including the Standard Model, as low-energy EFTs of some unknown fundamental theory.

Philosophers, too, are attracted by the idea that our current best theories might be best understood and formulated as EFTs. Some of them even claim that EFTs bring new insights into the way we understand the structure of the world, the relation between theories, and the ultimate aspirations of physics. Yet, how revolutionary and distinctively new these insights are remains a controversial matter. We provide a critical survey of some of the main philosophical debates over the implications of EFTs for our understanding of ontology (Sections 2 and 3), reduction (Section 4), and fundamentality (Section 5). In large part, these three philosophical interrogations go back to an early study of EFTs by Cao and Schweber [11]. While we do not necessarily agree with the views expressed by these authors, we recognize that their work has shaped many of the subsequent debates in the philosophical literature. Following Cao and Schweber, we structure the review around three questions:

1. Do EFTs underpin a specific ontological picture of the world or are they merely instruments to gain information about poorly understood domains?

2. Do EFTs support an anti-reductionist view of physics?

3. Do EFTs provide reasons to give up the ultimate aspiration of formulating a final and complete physical theory?

\section{$2 \quad$ A Different Picture of the World?}

Cao and Schweber claim that in the context of QFT, the framework of EFTs underwrites a pluralistic picture of the world arranged into an infinite hierarchy of quasi-autonomous domains delimited by wellseparated and physically meaningful mass scales. Each EFT (or set of EFTs) specifies a domain with its own set of entities, structures, and laws. Cao and Schweber rely on three main results and sets of techniques in QFT to support this claim: (i) the mechanism of spontaneous symmetry breaking (SSB); (ii) Wilsonian RG methods; (iii) the decoupling theorem. The latter is particularly important and will be the focus of this section. The relevance of SSB and RG methods for the question of autonomy has been discussed elsewhere $[2,3,5,6,17,23,32,38,47,49]$.

Broadly speaking, the decoupling theorem is a general result to the effect that the high-energy contributions to observables derived from specific types of QFTs have a negligible effect on the low-energy contributions to observables obtained by restricting these theories to low-energy regimes [1]. More precisely, one starts with the perturbative 
Lagrangian formulation of a renormalizable QFT containing a heavy and a light field characterized by scales $M$ and $m$, respectively, with $m \ll M$ (the light field does not need to be massive). If one eliminates the heavy field from the theory and defines a low-energy effective theory containing only the light field, the only manifestation of the heavy field is encoded in a finite number of perturbatively renormalizable parameters and wave-function renormalization factors, as well as in an infinite set of irrelevant operators suppressed by negative powers of the mass of the heavy field ("quasi-autonomy").

Cao and Schweber draw from the decoupling theorem the following lesson:

... with the decoupling theorem and the concept of EFT emerges a hierarchical picture of nature offered by QFT, one that explains why the description at any one level is so stable and is not disturbed by whatever happens at higher energies, and thus justifies the use of such descriptions. [11]

In other words, if the world contains a hierarchy of fields with sufficiently separated characteristic scales, the decoupling theorem offers a general justification for believing that the 'parts' of the system across scales are relatively insensitive from one another, isolating fields from each other, and offering a separate and distinct description of the system at each level.

The main response to this claim in the literature has been negative, because the decoupling theorem only applies to QFTs under highly restrictive conditions. As Hartmann points out early on [27], the highenergy theory needs to be perturbatively renormalizable, which sits in tension with the scenario of a never-ending tower of non-renormalizable EFTs. Yet, Hartmann remains somewhat elusive about the exact nature of this restriction. Perhaps Cao and Schweber assume that QFTs in particle physics are 'approximately' perturbatively renormalizable. Typically, for sufficiently low energies, non-renormalizable QFTs take the form of perturbatively renormalizable theories in the infinite cut-off limit. In Hartmann's defense, we note that there are clear exceptions: namely, theories for which all of the renormalizable interactions vanish because of symmetry constraints (e.g., the EFT Lagrangian describing the scattering of photons off atoms at low energies, chiral perturbation theory). These QFTs, insofar as one takes them to be interacting theories, are not approximately renormalizable and the decoupling theorem does not apply to them.

Bain provides a further defense of Cao and Schweber's argument [2]. He insists, following Georgi [21], on making a distinction between Wilsonian and continuum EFTs. Broadly speaking, this distinction is based on whether the split between the low-energy and high-energy physics depends on the mass parameter of the theory. Typically, a Wilsonian EFT is defined by integrating out heavy fields in the path integral formulation of a more comprehensive Lagrangian QFT. A continuum EFT is defined by selecting a relevant set of low-energy degrees of freedom, including all possible local operators compatible with the original structure of the Lagrangian and renormalizing the theory 
through dimensional regularization. The particular scaling behavior of the parameters of these operators is fixed by identifying, under some reasonable matching conditions, the correlation functions of the lowenergy theory with those of a higher energy theory. This identification occurs at a specific energy scale.

Bain's argument is then as follows. In the case of Wilsonian EFTs, one needs to assume that the high-energy theory is renormalizable in order to rely on the decoupling theorem and therefore faces the issues raised by Hartmann. In the case of continuum EFTs, by contrast, one does not need to make such an assumption. Strictly speaking, the decoupling theorem does not apply in this case. Yet, Bain claims that it is "inserted by hand in the form of matching conditions" [2]. How exactly the matching conditions might play the role of the decoupling theorem is not very clear. Surely, Bain means that if one constructs a continuum EFT in the sense specified above, then it displays the same kind of limited sensitivity to high-energy physics as stated in the decoupling theorem. And this feature warrants, in Bain's view, the claim that continuum EFTs are "capable of supporting an ontology of quasi-autonomous domains".

Bain's solution seems intuitive but it begs a number of questions. The decoupling theorem is not added "by hand" in the matching procedure; one merely observes the same kind of insensitivity as stated in the decoupling theorem and this is far from providing the sort of theoretical justification that Cao and Schweber seek in the first place. It is not enough to observe the existence of a good degree of insensitivity between successive domains on a case-by-case basis; to support their hierarchical picture, Cao and Schweber wish to underwrite these particular features of EFTs with a mathematically rigorous and general result. While the decoupling theorem seems unable to fulfill this task, Bain's attempt to trade the explanatory value and scope of a general result for a merely heuristic foundation also appears to be insufficient.

Our response, contra Bain, is to keep looking for a mathematical result but also to side with Hartmann in his critique of the use of the decoupling theorem. Since this theorem is unable to provide a sufficiently general ground for Cao and Schweber's picture, the only remaining option for justifying relative autonomy of scales seems to be an appeal to the formal features of the Wilsonian RG: e.g., the difference of scaling behavior between relevant and irrelevant local operators. In particular, contributions from irrelevant operators "dwindle" at low energies, to use Butterfield and Bouatta's felicitous expression [10]. Continuum EFTs then 'satisfy' the decoupling theorem for two main reasons: (i) in four dimensions, continuum EFTs contain a finite number of relevant operators and an infinite number of irrelevant operators; (ii) continuum EFTs contain an infinite number of free parameters. Point (i) means that the sensitivity to high-energy physics at low energies depends mainly on a finite number of independent parameters; point (ii), that it is always possible to match exactly the predictions of the low-energy theory with those of the high-energy theory and make sure that no sensitivity to high energies is left out. This suggests that in all 
situations the success of Cao and Schweber's argument about quasiautonomous domains may not rest on the validity of the decoupling theorem but on the success of Wilsonian RG methods. When the latter is considered, however, one faces a whole new set of issues beyond the scope of this review; most importantly, the problem of naturalness $[4,24,40,47,49]$.

\section{Instrumentalism and realism}

The reliance on what is done 'by hand', which has taken in the previous section the allure of a conceptual argument, suggests a more pragmatic and instrumentalist attitude towards EFTs: EFTs are efficient tools to derive precise predictions and organize physical effects according to natural hierarchies of scales. Maybe there is nothing more to EFTs than this instrumental and epistemic aspect, i.e., EFTs do not underwrite a particular picture of the world. This view has been discussed in the literature for at least as long as Cao and Schweber's competing argument.

For instance, Huggett and Weingard take a cautious stance motivated by the uncertainty about the existence of a final theory [29]. Since no course of future events is excluded by the EFT framework, they suggest that the most reasonable attitude is to take EFTs as scientific tools which are helpful for making progress in physics. One should not draw conclusions about the hierarchical structure of the world, the existence of a fundamental level, or the prospects of a completely unified theoretical description; the only philosophically sensible conclusion is that EFTs help to explore new domains and extend the scope of physics.

Hartmann, too, notes that EFTs do not force us to stop thinking about final theories [27]. Even if the project of constructing a final theory proves to be illusory at the end of the day, it still works as a fruitful regulative ideal. Like Huggett and Weingard, he finds a pragmatic position advocated by Georgi [21] to be the most attractive both epistemically and methodologically: the main reason to use EFTs is because they provide efficient tools to expand our knowledge. Hartmann goes further to suggest that EFTs fulfill a distinctive function alongside phenomenological models and putatively fundamental theories. This function should be appreciated rather than dismissed: it would be damaging to the progress of physics to eliminate scientific theorizing via EFTs on the grounds that they do not reveal reality or the true nature of the physical world.

Butterfield continues in a similar vein: the EFT approach is based on "an opportunistic or instrumentalist attitude to being unconfident" about the applicability of QFT at high energies [9]. This attitude has also been proposed by one of the authors in a previous work [23]. New empirical facts may become available, restore confidence and lead to a new and better physical theory. Furthermore, if the next physical theory happens not to use QFT methods, then the privileged status of the current best EFTs would be downgraded to that of mere instru- 
ments restricted to exploring a specific 'local' region. When or if this happens, then EFTs, with respect to their relevance for engaging with fundamental questions, would at best stay as monuments to physicists' perseverance.

This, of course, does not mean that EFTs do not possess important epistemic, pragmatic and computational virtues qua scientific instruments. In particular, they offer: (i) a local and intuitive understanding of low-energy phenomena, via simplified descriptions obtained by selecting only relevant degrees of freedom; (ii) efficient tools to solve computational problems, e.g., obtaining higher order correction terms; (iii) a direct solution to mathematical issues, e.g., the problem of ultraviolet divergences and consistency in QFT; (iv) efficient techniques to discover new physics and make new predictions.

In its most extreme form, the instrumentalist approach to EFTs takes them to be merely efficient tools which do not provide any ontological guidance about the world. This approach is often adopted as an instantiation of one's overall anti-realist stance. But even a scientific realist may find it tempting due to a certain psychological discomfort: EFTs are not meant by construction to disclose fundamental truths. As Shankar vividly puts it: "Often the opponents of EFT or even its practitioners feel they are somehow compromising" [42]. This contrasts with a more optimistic outlook about the ontological significance of EFTs: purportedly, EFTs are not simply efficient tools but also occupy a privileged position if one is to give the most accurate picture of the world by means of models currently thought to be successful. The difference between these viewpoints might at first seem vanishingly small but it actually reflects a significant philosophical disagreement in the debate over scientific realism.

Simply put, scientific realism is the stance that takes our best scientific theories to offer approximately true descriptions of the world. The history of science shows, however, that many past theories have proven to make radically false claims about unobservable entities and structures when these theories are assessed relative to their successors. This suggests that our current best theories might be in the same situation. The most popular response to this problem is a position called 'selective realism': even though our best theories do not get everything right, they still contain parts that are likely to remain (approximately) true [30, 36, 50]. Williams [48] and J. Fraser [19] have recently defended this position in the context of QFT: they argue that Wilsonian RG methods provide 'local' (i.e., restricted to QFT) tools to distinguish essential parts in current EFTs that are likely to withstand future theory change and give (approximately) true descriptions of the world. In particular, RG methods give selective realists some confidence in the claim that the low-energy content of the presently most successful EFTs is largely independent of the high-energy content of future theories and is therefore likely to remain unaffected by the discovery of new high-energy physics.

As this example shows, one needs to specify, methodologically speaking, the mechanism of selection implied in the concept of selective re- 
alism. Selection operates in a clear and consensual way if the complete theory is at hand: parts of EFTs that are likely to remain (approximately) true are then clearly identifiable as limit cases or specific approximations of the high-energy theory. In the case of bottom-up EFTs, however, no knowledge of a complete theory is available, and this might generate important disagreements on the selection of theoretical parts that are likely to survive. Various tricks may help to identify such parts, e.g., a relational analysis of EFTs with respect to each other. In order to remove the possibility of disagreement due to an excessively subjective assessment of what is real, the proponents of selective realism often rely on mathematical criteria and attribute theoretical longevity to the most invariant parts of the theory, for example, to the universality of low-energy dynamics specified by an infrared fixed point. If individual terms in the Lagrangians do not survive after theory change, universal dynamical properties have a better chance to find a place in the new theory.

The ambition of selective realism is to enable realists to make reliable ontological commitments about the world. Hence, besides selecting relevant theoretical parts, the selective realist also needs to say what these parts represent in the world. Williams makes a first step in this direction by criticizing the standard realist interpretative strategy: it "has led philosophers astray" in the context of QFT [48]. In particular, some philosophers highly concerned with the infamous mathematical issues plaguing realistic QFTs have been drawn to believe that mathematically rigorous yet highly unrealistic QFT models in lower dimensions give us more reliable ontological information about the world than the heuristic yet empirically adequate QFT models in high-energy physics. For instance, D. Fraser draws on the algebraic program in QFT to argue that QFT does not support an ontology of particles [18]. The framework of EFTs, Williams suggests in response, provides a more reliable and informed way to engage with the interpretation of physical theories: (i) interpret the content of theories insofar as they are successfully applied in physicists' practice; (ii) restrict the scope of interpretation to limited domains where theories are likely to remain approximately true; (iii) interpret theories in relation with one another and not as if they were putatively fundamental theories.

Williams's and J. Fraser's points are well taken. Clearly, the framework of EFTs provides a refinement of the notion of approximate truth by giving a mathematical specification of the limit at which a theory is likely to break down. The relational analysis of theories helps to identify their parts that are likely to survive theory change, e.g., via the application of RG methods. Universality arguments show that the current descriptions of low-energy physics are robust under variations of the high-energy content of a large class of potentially new theories.

This last point recently provoked an objection from Ruetsche [41]. A problem she identifies is that we do not know whether the future theory will fall within the bounds of RG analysis. If it does not, then the universality and robustness arguments will not work anymore. If it does by default, i.e., if the RG-space is not specified or supposed to 
be the space of all possible theories, then we lose the confidence gained by the concrete implementation of RG methods. Ruetsche makes another relevant point: RG enthusiasts are often quite vague about the features they take EFTs to latch onto. And without an account of the specific ontological guidance EFTs provide in currently accessible domains, Williams's and J. Fraser's defense of selective realism remains too open-ended. One might focus on invariant higher-order structural features exhibited by the mathematical structure of Lagrangian EFTs and of the RG space. Yet, Ruetsche's point would still apply. If the formalism of the new theory after a future theory change happens to be incommensurable with the mathematical structure of QFT, even the parts favored by selective realists will become void of ontological status. While this prospect must always be kept in mind, we believe that, as of today, selective realism still provides a welcome alternative to the instrumentalist approach to EFTs. Yet, the selective realist needs to say explicitly what the world is made of in currently accessible domains.

\section{Reduction and Emergence}

While many physicists believe that the men and women of their profession ought to seek for fundamental truths about nature, those following the EFT approach spend their time and effort on a model unfit by its very design to reveal any such truth. The status of the fundamental, let alone ultimate, physical theory in the light of EFTs will be our subject for the remainder of this article.

If one assumes that a fundamental theory exists, it is an open question whether one can derive EFTs from this fundamental theory under some plausible assumptions. It is also an open question whether EFTs can be derived from each other. While these two interrogations remain distinct, many physicists agree that such derivations are possible in both situations. By agreeing, they adopt a reductionist perspective on EFTs. Giudice, for example, writes: "Effective field theories are a powerful realization of the reductionist approach" [22].

The topic of reduction is highly controversial and it is far beyond the scope of this review to do justice to the rich discussions that have animated the philosophical literature in the last decades. In the context of physics, it is relatively standard to distinguish between conceptual, explanatory, and ontological reduction. Conceptual and explanatory reducibility is taken as a guide to ontological reducibility. The idea, in a nutshell, is the following. Suppose that all the concepts used to specify the entities at some level $L$ can be translated in terms of, or replaced by, the concepts used to specify the entities at some more fundamental level $L^{\prime}$. Suppose in addition that the law-like behavior of the entities at level $L$ can be fully explained in terms of the law-like behavior of the entities at level $L^{\prime}$. Then, there are good reasons to believe that the entities at level $L$ are reducible to the entities at level $L^{\prime}$. Here, reduction can be understood either in an eliminative or in a conservative sense: 
1. If the descriptions at levels $L$ and $L^{\prime}$ are radically incompatible with one another, there are good reasons to eliminate the entities at level $L$ altogether;

2. If the descriptions at level $L$ approximate those at level $L^{\prime}$, there are good reasons to identify the entities at level $L$ with some composite of the entities at level $L^{\prime}$, say, a complex pattern constituted by the behavior of those entities.

Emergence is traditionally understood in terms of irreducibility: the behavior and the properties of some entities at level $L$ are emergent if they cannot be reduced to the behavior and properties of entities at some level $L^{\prime}$.

Nagel famously proposed a relatively simple scheme for analyzing conceptual and explanatory reduction [33]. The basic idea is to focus on logico-linguistic relations between theories. A higher-level and less fundamental theory $T_{t}$ reduces to a lower-level and more fundamental theory $T_{b}$ if and only if the laws of $T_{t}$ can be deduced from the laws of $T_{b}$ with "bridge laws" relating the vocabulary of the two theories and some auxiliary assumptions, if necessary. Most philosophers agree that, in general, it is sufficient to derive an approximate version of $T_{t}$ in order to explain away its success. It is also worth noting that the philosophers' lexicon differs somewhat from that of the physicists: philosophers say that $T_{t}$ reduces to $T_{b}$ if $T_{t}$ can be shown to be a part of $T_{b}$, while physicists often say that $T_{b}$ reduces to $T_{t}$ if $T_{b}$ simplifies to $T_{t}$ in the relevant limit.

Back to EFTs, Cao and Schweber defend an anti-reductionist view. They base their argument on the central claim that RG methods do not by themselves determine the complexity and novelty of low-energy domains without empirical inputs [11]. Cao and Schweber acknowledge the existence of 'causal' connections between different domains: the decoupling theorem and Wilsonian RG methods give a precise description of the effects of the high-energy physics on the dynamics of the low-energy physics. However, they argue that these causal connections are not sufficient to exhaust the theoretical content of the low-energy effective theory. Here, again, they remain somewhat elusive. Their argument seems to follow from the claim that, typically, the appropriate structure of the low-energy degrees of freedom, the form of their dynamics, and the value of the couplings cannot be simply derived from the high-energy dynamics but are instead determined in large part by appealing to low-energy empirical information.

Castellani [12] and Hartmann [27] give a more cautious assessment. They note that, in many cases, distinct EFTs at different levels can be explicitly related by means of deductive relations and bridge laws. Here, the clearest example is provided by the Wilsonian derivation of a low-energy theory from a high-energy one by integrating out heavy degrees of freedom. And this suggests that, at least in principle, the framework of EFTs does not exclude a full reconstruction of low-energy EFTs from a more fundamental high-energy theory and therefore remains neutral with respect to reductionism and antireductionism.

More recently, Bain made an attempt to extend further Cao and 
Schweber's initial position by highlighting two crucial features of concrete cases of EFTs [2,3]. Firstly, in most realistic cases, the low-energy dynamical equations and degrees of freedom are formally distinct from those of the high-energy theory. For instance, two successive theories typically exhibit distinct structural properties (e.g., the symmetries of their Lagrangian). And if the difference between the two theories and their degrees of freedom is "substantial enough", Bain claims, the theories are not, strictly speaking, a "part" of one another and admit distinct ontological interpretations [2]. We agree in principle: for instance, the expression of the pion fields is substantially distinct and not directly derivable from the expression of the quark and gluons fields. However, taken by itself, Bain's criterion of "substantial" formal distinctness does not pose a real threat to the existence of a successful Nagelian reduction. For one thing, it does not prevent the possibility of mapping the low-energy and high-energy degrees of freedom on one another. For another, a successful Nagelian reduction only requires the low-energy theory to be similar to, but not formally identical with, a 'part' of the high-energy theory.

Secondly, the relation between two EFTs involve approximations and heuristic reasoning: e.g., performing a saddle point approximation when integrating out heavy fields or taking the zero mass limit of some light field. Bain claims that these features make the task of formulating the relation between two theories in terms of a mathematical derivation difficult, if not impossible. Here, again, successful Nagelian reductions are relatively permissive to the use of intermediary approximations, assumptions, and heuristic reasoning. The ultimate goal of a Nagelian reduction is to explain away the success of the low-energy theory by means of the high-energy theory, not to provide a strict derivation relying only on the resources of the high-energy theory.

Butterfield offers a further response both to Cao and Schweber and to Bain [9]. He centers his defense of Nagelian reduction between successive EFTs around one central idea: RG methods specify families of Nagelian reductions of low-energy (approximately) renormalizable EFTs to high-energy non-renormalizable ones. Butterfield does not say much about the potential complexity of the tower of EFTs but his point is relatively straightforward to understand:

1. Nagelian reductions hold for low-energy EFTs obtained by integrating out high-energy degrees of freedom;

2. Nagelian reductions hold when we run the RG flow towards low energies and if we make an approximation, e.g., by eliminating negligible non-renormalizable terms;

3. Nagelian reductions hold when we construct EFTs in the bottomup method and connect them to higher theories in the RG space by means of matching conditions (the reduction demands in this case a few more approximations).

Furthermore, Butterfield suggests a concept of emergence compatible with successful Nagelian reductions $[7,8]$. The behavior of an entity is emergent when it displays novel and robust properties with respect to 
some appropriate comparison class, for instance, the class of its subatomic components. 'Novelty' means that the low-energy description of a theory displays distinctive features which are not present in the high-energy description. 'Robustness' means that we can modify the high-energy description of a theory and still observe invariant novel features at low energies. RG methods provide tools to both exhibit these features across scales and establish approximate deductive relations between low- and high-energy theories.

His optimism notwithstanding, Butterfield seems to be too confident about the ability of a high-energy theory to explain away the phenomena described by a low-energy theory via RG methods, when the two theories are not simply related in the Wilsonian sense. On the one hand, chiral perturbation theory gives a good example of a low-energy EFT which cannot be directly matched to its high-energy theory by means of perturbative calculations. Lattice QFT methods may provide some relief here, but it remains a remarkable fact that there exists no energy regime enabling a direct comparison between the perturbative quantities obtained from chiral perturbation theory and the perturbatively renormalizable version of QCD. More generally, the phenomenon of confinement in the low-energy regime of QCD suggests that the law-like behavior of quarks and gluons cannot even in principle be used to explain the law-like behavior of hadrons. On the other hand, the success of Butterfield's RG-based argument depends on considerations of naturalness: the sensitivity of relevant operators to high energies goes some way against the robustness of low-energy phenomena [47].

\section{Conclusion: Fundamentality and the ul- timate aspirations of physics}

Effective field theories are not fundamental by intent and by design. Physicists may be embarrassed by this state of affairs but they are also quick to recognize the importance of the EFT framework for computing the values of physical observables in a situation when no such calculation is feasible in the high-energy theory. We discussed in the previous section whether these kinds of concerns prevent a reductionist view on the inter-theoretic relations between putatively fundamental theories and EFTs, and between EFTs themselves. There exists, however, a more general meaning of reduction which might be seen as making the question of inter-theoretic reduction between successive theories somewhat less urgent. We conclude by discussing this meaning, for it also relates to two distinctive visions of the future of physics.

In its most extreme form, the reductionist approach is based on the claim that, ultimately, all non-fundamental theories in physics will be reduced to a single unified, complete, and final theory. Cao and Schweber argue that the EFT framework goes some way towards undermining our confidence in the existence and relevance of such a theory. Instead, this framework favors the scenario of a never-ending tower of 
EFTs with no ultimate or overarching theoretical account that would unify them or provide a single, fundamental description of the world.

The elusive and highly speculative character of this argument has made it an easy target in the literature [9, 12, 13, 27, 29, 39]. Overall, the main response is that the framework of EFTs does not warrant the scenario of a never-ending tower of EFTs as opposed to other possibilities, for two principal reasons:

1. We may either find the final and complete theory or keep replacing current EFTs by more comprehensive ones;

2. The fact that current realistic QFTs are best understood and formulated as EFTs does not imply anything specific about the existence or the form of a final theory.

As Butterfield summarizes it, "... in the present state of knowledge, we have no compelling reason, even for energies for which we can be confident of the QFT framework (and so: independently of considerations of quantum gravity), to believe in what ... I called the 'vision', namely a tower of theories that are not each derivable from some single theory, as an approximation describing physics within their own energy range" $[9]$.

These last remarks suggest a number of general lessons from the debate on EFTs. Firstly, few philosophers and physicists take seriously the scenario of a never-ending tower of EFTs, although it is an interesting and conceptually challenging picture which finds some grounds in low-energy physics. But it is overly speculative. Secondly, among the rare advocates of this picture, 'effective theory' is taken to refer to any kind of non-fundamental, approximate theory. The view is also typically inferred from the repeated failure of our best putatively fundamental theories over time:

Unlike perhaps in decades and centuries gone by, no competent scientist should retain an unfailing commitment to any theory. All theories are incomplete, even given that some theories are better than others. ... Finally, one of the most profound shifts in our thinking over the decades is that it is really no longer appropriate to speak of 'the correct theory.' There is no correct theory. Our tasks are to improve theories via the effective theory approach, to seek deeper and simplifying assumptions that account for it, solidify those into a new theory, and then treat that new theory as an effective theory, and repeat. ... Theories are never to be trusted - they are always "wrong" in the end - and with concerted effort we can even anticipate when and how they will break down. [45]

One should be cautious about making the notion of effective theory too open-ended and too inclusive when drawing these kinds of inferences. The final theory, if any, might either emerge as the ultimate completion of a long and lasting theoretical endeavor or as a singular revolutionary theoretical event in sharp discontinuity with what had been done previously. In this context, pessimistic conclusions from the 
past are neither the only nor necessarily the most successful way to draw philosophically interesting lessons.

Finally, a more methodological point: for sure, aversion to epistemic risk warrants some sympathy towards the 'tower of EFTs' scenario. Only those willing to take ontological bets will continue to point in the direction of a complete and final theory. This situation has not changed much since the 1990s, when the philosophical debate on EFTs began following the work of Cao and Schweber [11]. Although their original arguments have been variously criticized, refuted, elaborated, or re-established by different authors, the conceptual palette has remained similar overall. To be sure, there are some novelties. For instance, the recent attempts to defend the interpretative and epistemic virtues of EFTs by means of a careful examination of their mathematical structure are well-received. Effective field theories hold good prospects for asking ontologically perspicuous and sensible questions about currently accessible domains. Yet, we believe that, as of today, they do not give good enough reasons to draw a definitive conclusion about more fundamental philosophical questions. Meanwhile, the framework of EFTs still remains an indispensable instrument for conducting fruitful scientific research.

\section{Acknowledgements}

We warmly thank Thomas Duguet and his colleagues at the Espace de Structure Nucléaire Théorique for many interesting discussions on the topics of this review.

\section{References}

[1] T. Appelquist and J. Carrazone. Infrared singularities and massive fields. Phys. Rev. D, 11:2856-2861, 1975.

[2] J. Bain. Effective field theories. In R. Batterman, editor, The Oxford Handbook of Philosophy of Physics, pages 224-254. Oxford University Press, Oxford, 2013.

[3] J. Bain. Emergence in effective field theories. European Journal for Philosophy of Science, 3(3):257-273, 2013.

[4] J. Bain. Why be natural? Foundations of Physics, forthcoming.

[5] R. W. Batterman. Emergence, singularities, and symmetry breaking. Foundations of Physics, 41(6):1031-1050, 2011.

[6] R. W. Batterman. Autonomy of theories: An explanatory problem. Nous, 52(4):858-873, 2018.

[7] J. Butterfield. Emergence, reduction and supervenience: A varied landscape. Foundations of Physics, 41:920-959, 2011.

[8] J. Butterfield. Less is different: Emergence and reduction reconciled. Foundations of Physics, 41:1065-1135, 2011. 
[9] J. Butterfield. Reduction, emergence and renormalization. Journal of Philosophy, 111:5-49, 2014, arXiv:1406.4354.

[10] J. Butterfield and N. Bouatta. Renormalization for philosophers. In T. Bigaj and C. Wuthrich, editors, Metaphysics in Contemporary Physics, pages 437-485. Brill Rodopi, Leiden, 2015. arXiv:1406.4532.

[11] T. Y. Cao and S. Schweber. The conceptual foundations and the philosophical aspects of renormalization theory. Synthese, 97:33108, 1993.

[12] E. Castellani. Reductionism, emergence, and effective field theories. Studies in History and Philosophy of Science Part B, 33(2):251-267, 2002.

[13] K. Crowther. Effective spacetime. Understanding Emergence in Effective Field Theory and Quantum Gravity. Springer, Zurich, 2015.

[14] P. Dirac. The relation between mathematics and physics. Proceedings of the Royal Society (Edinburgh), 59:122-129, 1939. Quoted in [31, p. 277].

[15] H. Euler. Über die Streuung von Licht an Licht nach der Diracschen Theorie. Ann. der Physik, 418:398-448, 1936.

[16] H. Euler and B. Kockel. Über die Streuung von Licht an Licht nach der Diracschen Theorie. Naturwissenschaften, 23:246, 1935.

[17] A. Franklin. Whence the effectiveness of effective field theories? The British Journal for the Philosophy of Science, forthcoming.

[18] D. Fraser. The fate of 'particles' in quantum field theories with interactions. Studies in History and Philosophy of Science Part B, 39(4):841-859, 2008.

[19] J. D. Fraser. Renormalization and the formulation of scientific realism. Philosophy of Science, 85(5):1164-1175, 2018.

[20] M. Gell-Mann. The interpretation of the new particles as displaced charge multiplets. Il Nuovo Cimento, 4:848-866, 1956.

[21] H. Georgi. Effective quantum field theories. Annual Review of Nuclear and Particle Science, 43:209-252, 1993.

[22] G. F. Giudice. Naturally speaking: The naturalness criterion and physics and LHC. In G. Kane and A. Pierce, editors, Perspectives on LHC Physics, pages 155-178. World Scientific, Singapore, arXiv:0801.2562.

[23] A. Grinbaum. On the eve of the LHC: conceptual questions in high-energy physics. Technical report, CEA, 2008, arXiv:0806.4268.

[24] A. Grinbaum. Which fine-tuning arguments are fine? Foundations of Physics, 42:615-631, 2012.

[25] A. Grinbaum. How device-independent approaches change the meaning of physical theory. Studies in History and Philosophy of Science Part B, 58:22-30, 2017, arXiv:1512.01035. 
[26] A. Grinbaum. The effectiveness of mathematics in physics of the unknown. Synthese, 196:973-989, 2019, philsci/13191.

[27] S. Hartmann. Effective field theories, reductionism and scientific explanation. Studies in History and Philosophy of Science Part B, 32(2):267-304, 2001.

[28] W. Heisenberg and H. Euler. Folgerungen aus der Diracschen Theorie des Positrons. Z. Phys., 98:714, 1936.

[29] N. Huggett and R. Weingard. The renormalisation group and effective field theories. Synthese, 102:171-194, 1995.

[30] P. Kitcher. The Advancement of Science: Science Without Legend, Objectivity Without Illusions. Oxford University Press, 1993.

[31] H. Kragh. Dirac: A Scientific Biography. Cambridge University Press, 1990.

[32] M. Morrison. Emergent physics and micro-ontology. Philosophy of Science, 79:141-166, 2012.

[33] E. Nagel. The Structure of Science: Problems in the Logic of Scientific Explanation. Harcourt, Brace \& World, 1961.

[34] H. P. Noyes, editor. Proceedings of the Fourth Annual Rochester Conference on High Energy Nuclear Physics. The University of Rochester and The National Science Foundation, 1954.

[35] A. Pich. Effective field theory. In R. Gupta et al., editors, Proceedings of Les Houches Summer School of Theoretical Physics 'Probing the Standard Model of Particle Interactions', volume II, page 949, Amsterdam, 1999. Elsevier, arXiv:hep-ph/9806303.

[36] S. Psillos. Scientific Realism: How Science Tracks Truth. Routledge, 1999.

[37] W. Quine. Pursuit of Truth. Harvard University Press, 1992. Revised edition.

[38] S. Rivat. Renormalization scrutinized. Studies in History and Philosophy of Science Part B, forthcoming, philsci/16072.

[39] D. Robinson. Renormalization and the effective field theory programme. In D. Hull et al., editors, Proceedings of the PSA, pages 393-403, 1992.

[40] J. Rosaler and R. Harlander. Naturalness, Wilsonian renormalization, and "fundamental parameters" in quantum field theory. Studies in History and Philosophy of Science Part B, 66:118-134, 2019.

[41] L. Ruetsche. Renormalization group realism: The ascent of pessimism. Philosophy of Science, 85(5):1176-1189, 2018.

[42] R. Shankar. Effective field theory in condensed matter physics. In T. Y. Cao, editor, Conceptual Foundations of Quantum Field Theory, pages 47-55. Cambridge University Press, Cambridge, 1999. 
[43] S. Weinberg. What is quantum field theory and what did we think it was. In T. Y. Cao, editor, Conceptual Foundations of Quantum Field Theory, pages 241-251. Cambridge University Press, Cambridge, 1999.

[44] S. Weinberg. Effective field theory, past and future. International Journal of Modern Physics A, 31(06):1630007, 2016.

[45] J. D. Wells. Effective Theories in Physics: From Planetary Orbits to Elementary Particle Masses. Springer, Geneva, 2012.

[46] E. Wigner. The unreasonable effectiveness of mathematics in the natural sciences. Communications in Pure and Applied Mathematics, 13:1-14, 1960.

[47] P. Williams. Naturalness, the autonomy of scales, and the 125 GeV Higgs. Studies in History and Philosophy of Modern Physics, 51:82-96, 2015.

[48] P. Williams. Scientific realism made effective. The British Journal for the Philosophy of Science, 70(1):209-237, 2019.

[49] P. Williams. Two notions of naturalness. Foundations of Physics, forthcoming, philsci/15347.

[50] J. Worrall. Structural realism: The best of both worlds? Dialectica, 43(1-2):99-124, 1989. 